\title{
Semistability of Principal Bundles on a Kähler Manifold with a Non-Connected Structure Group
}

\author{
Indranil BISWAS ${ }^{\dagger}$ and Tomás L. GÓMEZ $\ddagger$ \\ $\dagger$ School of Mathematics, Tata Institute of Fundamental Research, \\ Homi Bhabha Road, Bombay 400005, India \\ E-mail: indranil@math.tifr.res.in \\ ¥ Instituto de Ciencias Matemáticas (CSIC-UAM-UC3M-UCM), \\ Nicolás Cabrera 15, Campus Cantoblanco UAM, 28049 Madrid, Spain \\ E-mail: tomas.gomez@icmat.es
}

Received October 29, 2013, in final form February 07, 2014; Published online February 12, 2014 http://dx.doi.org/10.3842/SIGMA.2014.013

\begin{abstract}
We investigate principal $G$-bundles on a compact Kähler manifold, where $G$ is a complex algebraic group such that the connected component of it containing the identity element is reductive. Defining (semi)stability of such bundles, it is shown that a principal $G$-bundle $E_{G}$ admits an Einstein-Hermitian connection if and only if $E_{G}$ is polystable. We give an equivalent formulation of the (semi)stability condition. A question is to compare this definition with that of [Gómez T.L., Langer A., Schmitt A.H.W., Sols I., Ramanujan Math. Soc. Lect. Notes Ser., Vol. 10, Ramanujan Math. Soc., Mysore, 2010, 281-371].
\end{abstract}

Key words: Einstein-Hermitian connection; principal bundle; parabolic subgroup; (semi)stability

2010 Mathematics Subject Classification: 53C07; 14F05

\section{Introduction}

Let $X$ be a compact connected Kähler manifold equipped with a Kähler form $\omega$. Let $G$ be a connected complex reductive group. The connected component, containing the identity element, of the center of $G$ will be denoted by $Z_{0}(G)$. In the study of principal $G$-bundles on $X$, it is usually assumed that the group $G$ is connected.

In [8], Ramanathan gave the following definition. A principal $G$-bundle $E$ is stable (respectively, semistable) if for all proper parabolic subgroups $P$ and all reductions of structure group $\left.E_{P} \subset E\right|_{U}$ on a big open subset $U \subset X$ (complement of a Zariski closed subset of codimension at least two), and for all strictly dominant characters $\chi: P \longrightarrow \mathbb{C}^{*}$, the associated line bundle $E_{P}(\chi)$ over $U$ satisfies the inequality

$$
\left.\operatorname{deg}\left(E_{P}(\chi)\right)<0 \quad \text { (respectively, } \operatorname{deg}\left(E_{P}(\chi)\right) \leq 0\right),
$$

where the degree is calculated with respect to the Kähler form $\omega$. We recall that a strictly dominant character of $P$ is a character of $P$ trivial on $Z_{0}(G)$ such that the dual of the line bundle on $G / P$ associated to the character is ample.

A reduction $\left.E_{P} \subset E\right|_{U}$ on a big open set $U \subset X$ to a parabolic subgroup $P$ is called admissible if for every character $\chi$ of $P$ trivial on $Z_{0}(G)$, we have $\operatorname{deg}\left(E_{P}(\chi)\right)=0$. A semistable principal $G$-bundle on $X$ is called polystable if there exists a reduction $E_{L(P)} \subset E_{G}$ to a Levi factor $L(P)$ of a parabolic subgroup $P \subset G$ such that the following two conditions hold:

- the principal $L(P)$-bundle $E_{L(P)}$ is stable, and

- the reduction of $E_{G}$ to $P$ obtained by extending the structure group of $E_{L(P)}$ to $P$ using the inclusion $L(P) \hookrightarrow P$ is admissible. 
Ramanathan used this notion in $[9,10]$ to construct the moduli space of semistable principal bundles when $\operatorname{dim} X=1$. In [11] Ramanathan and Subramanian proved that a principal $G$ bundle admits an Einstein-Hermitian connection if and only if it is polystable.

Behrend defined stability for group schemes in [3]. A reductive group scheme $\mathcal{G} / X$ on $X$ is stable (respectively, semistable) if for all parabolic subgroup schemes $\mathcal{P} / X$, we have

$$
\operatorname{deg}(\mathcal{P} / X):=\operatorname{deg}(\operatorname{ad}(\mathcal{P}))<0 \quad \text { (respectively, } \operatorname{deg}(\operatorname{ad}(\mathcal{P})) \leq 0),
$$

where $\operatorname{ad}(\mathcal{P})$ is the associated Lie algebra bundle on $X$. Then he defines a principal $G$-bundle on $X$ to be stable (respectively, semistable) if the associated group scheme $\operatorname{Ad}(E)$ is semistable (respectively, semistable).

If $G$ is connected, then it is easy to see that Behrend's definition of stability coincides with Ramanathan's, because the normalizer $N_{G}(P)$ of a parabolic subgroup $P$ in $G$ is equal to $P$ when $G$ is connected.

If $G$ is reductive but not connected, the moduli space of principal $G$-bundles has been constructed in [5] for projective varieties. Given a 1-parameter subgroup $\lambda: \mathbb{C}^{*} \longrightarrow[G, G] \subset G$, a parabolic subgroup $P(\lambda)$ is defined as follows [12]

$$
P(\lambda)=\left\{g \in G: \lim _{t \rightarrow \infty} \lambda(t) g \lambda(t)^{-1} \text { exists in } G\right\}
$$

and the condition of stability is checked only with these parabolic subgroups. The construction in [5] is done using Geometric Invariant Theory, and one obtains a stability condition involving Hilbert polynomials. Looking only at the leading coefficients one obtains the associated "slope" stability, as usual, which only involves degrees. This is the stability we consider, since this is the one which is expected to correspond to the existence of Einstein-Hermitian connections.

Here we address the problem of finding a condition for the existence of an Einstein-Hermitian connection on a principal $G$-bundle on $X$ when $G$ is not connected.

If $G_{0}$ is the connected component of identity of $G$, then $E \longrightarrow E / G_{0}$ is a principal $G_{0}$-bundle on $Y:=E / G_{0}$, and $Y$ is a finite étale cover of $X$. In Section 2 we define the principal $G$-bundle $E$ to be polystable when the principal $G_{0}$-bundle $E \longrightarrow Y$ is polystable (cf. Definition 1), and prove that this is a necessary and sufficient condition for the existence of an Einstein-Hermitian connection on $E$. In Section 3 we show that this definition of polystability is equivalent to checking the usual condition for those parabolic subgroups of $G$ of the form $N_{G}(\mathfrak{p})$, where $\mathfrak{p}$ is a parabolic subalgebra of the Lie algebra $\mathfrak{g}$ of $G$. Note that $N_{G}(\operatorname{Lie}(P))=N_{G}\left(P^{0}\right)$ for any parabolic subgroup $P$, where $\operatorname{Lie}(P)$ is the Lie algebra and $P^{0}$ is the connected component of $P$ containing the identity element.

We remark that, if $G$ is connected, all parabolic subgroups are of this form because for a connected reductive group, $N_{G}(\mathfrak{p})=N_{G}(P)=P$.

It is natural to ask whether our condition is equivalent to the condition in [5] in terms of 1-parameter subgroups. For any parabolic subgroup $P$, it is $P \subset N_{G}(\operatorname{Lie}(P))$. Hence, a reduction of structure group to $P$ gives reduction to $N_{G}(\operatorname{Lie}(P))$, and therefore if a principal $G$-bundle is (semi)stable in the sense of [5], then it is also (semi)stable in our sense.

The implication in the other direction is not clear, since there exist examples of non-connected groups $G$ and 1-parameter subgroups $\lambda$ such that $N_{G}\left(P(\lambda)^{0}\right)$ is strictly larger than $P(\lambda)$. It would be interesting to be able to compare the two definitions.

\section{Connections on principal bundles}

\subsection{Semistable and polystable bundles}

Let $G$ be a reductive linear algebraic group defined over $\mathbb{C}$. We do not assume that $G$ is connected. Let $G_{0} \subset G$ be the connected component containing the identity element. We note 
that $G_{0}$ is a normal subgroup of $G$. The quotient group

$$
\Gamma:=G / G_{0}
$$

parametrizes the connected components of $G$.

Let $X$ be a compact connected Kähler manifold equipped with a Kähler form $\omega$. Let

$$
E_{G} \longrightarrow X
$$

be a holomorphic principal $G$-bundle on $X$. Consider the quotient map

$$
E_{G} \stackrel{\phi}{\longrightarrow} E_{G} / G_{0}=: Y \text {. }
$$

The natural projection

$$
f: Y \longrightarrow X
$$

is a unramified Galois covering map with Galois group $\Gamma$ (defined in (2)). The pulled back form $f^{*} \omega$ is a Kähler form on $Y$. It should be clarified that $Y$ need not be connected.

The projection $\phi$ in (3) makes $E_{G}$ a holomorphic principal $G_{0}$-bundle on $Y$.

Definition 1. The principal $G$-bundle $E_{G}$ on $X$ is called semistable (respectively, stable) if for each connected component $Y^{\prime}$ of $Y$, the principal $G_{0}$-bundle

$$
\phi^{-1}\left(Y^{\prime}\right) \longrightarrow Y^{\prime}
$$

is semistable (respectively, stable). Similarly, $E_{G}$ on $X$ is called polystable if the principal $G_{0^{-}}$ bundle $\phi^{-1}\left(Y^{\prime}\right) \longrightarrow Y^{\prime}$ is polystable for every connected component $Y^{\prime}$ of $Y$.

Lemma 1. A principal $G$-bundle $E_{G}$ on $X$ is semistable (respectively, polystable) if for some connected component $Y^{\prime}$ of $Y$, the principal $G_{0}$-bundle

$$
\phi^{-1}\left(Y^{\prime}\right) \longrightarrow Y^{\prime}
$$

is semistable (respectively, polystable). The same criterion holds for stability.

Proof. Take two connected components $Y_{1}$ and $Y_{2}$ of $Y$. Since the covering map $f$ in (4) is Galois, there is an element $g$ of the Galois group such that the automorphism $g$ of $Y$ takes $Y_{1}$ to $Y_{2}$. Let

$$
\tilde{g}:=\left.g\right|_{Y_{1}}: Y_{1} \longrightarrow Y_{2}
$$

be this isomorphism. Let $E_{1}$ (respectively, $E_{2}$ ) be the restriction of the principal $G_{0}$-bundle $E_{G} \longrightarrow Y$ to $Y_{1}$ (respectively, $\left.Y_{2}\right)$. Since $f \circ \widetilde{g}=f$, and $\operatorname{Lie}(G)=\operatorname{Lie}\left(G_{0}\right)$, we have

$$
\widetilde{g}^{*} \operatorname{ad}\left(E_{2}\right)=\operatorname{ad}\left(E_{1}\right) \text {. }
$$

On the other hand, a principal $G_{0}$-bundle is semistable (respectively, polystable) if and only if the corresponding adjoint vector bundle is semistable (respectively, polystable); see [1, p. 214, Proposition 2.10] and [1, p. 224, Corollary 3.8]. Therefore, from (5) we conclude that $E_{1}$ is semistable (respectively, polystable) if and only if $E_{2}$ is semistable (respectively, polystable).

This isomorphism in (5) is compatible with the Lie algebra structure of the fibers of the two adjoint bundles. We recall that a principal $G_{0}$-bundle $F_{G_{0}}$ is stable if for every parabolic subalgebra bundle $\widetilde{\mathfrak{p}} \subset \operatorname{ad}\left(F_{G_{0}}\right)$, we have

$$
\operatorname{degree}(\widetilde{\mathfrak{p}})<0
$$

(see [3]). Therefore, if $E_{1}$ is stable, then $E_{2}$ is also stable. 


\subsection{Einstein-Hermitian connections}

Any two maximal compact subgroups of $G$ differ by an inner automorphism of $G$. Fix a maximal compact subgroup $K \subset G$. The quotient $G / K$ is a contractible manifold, in particular, $G / K$ is connected.

Take a holomorphic principal $G$-bundle $E_{G}$ over $X$. A Hermitian structure on $E_{G}$ is a $C^{\infty}$ reduction of structure group of $E_{G}$ to $K$. Since $G / K$ is contractible, and any $C^{\infty}$ fiber bundle with a contractible fiber is trivial, it follows immediately that $E_{G}$ admits Hermitian structures.

Any two connections on the principal $G$-bundle $E_{G}$ differ by a smooth 1-form with values in $\operatorname{ad}\left(E_{G}\right)$. Two $C^{\infty}$ connections $\nabla_{1}$ and $\nabla_{2}$ on the principal $G$-bundle $E_{G}$ are called equivalent if $\nabla_{1}-\nabla_{2}$ is of type $(1,0)\left[7\right.$, p. 87]. The complex structure on the total space of $E_{G}$ defines an equivalence class of connections on $E_{G}[7$, p. 87, Proposition 2].

Let $E_{K} \subset E_{G}$ be a Hermitian structure. Then there is a unique connection $\nabla$ on the principal $K$-bundle $E_{K}$ such that the connection $\widetilde{\nabla}$ on $E_{G}$ induced by $\nabla$ lies in the equivalence class of connections defined by the complex structure on $E_{G}$ [2, pp. 191-192, Proposition 5]. This $\widetilde{\nabla}$ is called the Chern connection corresponding to $E_{K}$.

Let $\operatorname{ad}\left(E_{K}\right) \longrightarrow X$ be the adjoint vector bundle for the principal $K$-bundle $E_{K}$. Let

$$
\mathcal{K}(\nabla) \in \Omega^{1,1}\left(\operatorname{ad}\left(E_{K}\right)\right)
$$

be the curvature of the above connection $\nabla$ on $E_{K}$. The Hermitian structure $E_{K}$ is called Einstein-Hermitian if the section

$$
\Lambda_{\omega} \mathcal{K}(\nabla) \in \Omega^{0}\left(\operatorname{ad}\left(E_{K}\right)\right)
$$

is given by some element of the center of the Lie algebra of $K$; here $\Lambda_{\omega}$ is the adjoint of multiplication of differential forms by the Kähler form $\omega$.

Theorem 1. A holomorphic principal $G$-bundle $E_{G}$ on $X$ admits an Einstein-Hermitian structure if and only if $E_{G}$ is polystable.

Given a polystable principal $G$-bundle $E_{G}$ over $X$, the Chern connection on $E_{G}$ corresponding to a Einstein-Hermitian structure on $E_{G}$ is independent of the choice of Einstein-Hermitian structure.

Proof. First assume that $E_{G} \longrightarrow X$ admits an Einstein-Hermitian structure. The connection on the adjoint vector bundle $\operatorname{ad}\left(E_{G}\right)$ induced by an Einstein-Hermitian connection on $E_{G}$ is also Einstein-Hermitian. Therefore, $\operatorname{ad}\left(E_{G}\right)$ is polystable. Take any connected component $Y^{\prime}$ of $Y$. Let $\bar{f}$ be the restriction of $f$ to $Y^{\prime}$. Let $E^{\prime} \longrightarrow Y^{\prime}$ be the principal $G_{0}$-bundle obtained by restricting $E_{G} \longrightarrow Y$ to $Y^{\prime}$.

Since $\bar{f}^{*} \operatorname{ad}\left(E_{G}\right)=\operatorname{ad}\left(E^{\prime}\right)$, and $\operatorname{ad}\left(E_{G}\right)$ is polystable, we conclude that $\operatorname{ad}\left(E^{\prime}\right)$ is polystable [4, p. 439, Proposition 2.3]. Hence $E^{\prime}$ is polystable [1, p. 224, Corollary 3.8].

To prove the converse, assume that the principal $G_{0}$-bundle $E_{G} \longrightarrow X$ is polystable. Take a connected component $Y^{\prime}$ of $Y$. As before, $\bar{f}$ is the restriction of $f$ to $Y^{\prime}$, and $E^{\prime} \longrightarrow Y^{\prime}$ is the principal $G_{0}$-bundle obtained by restricting $E_{G} \longrightarrow Y$ to $Y^{\prime}$. The adjoint vector bundle ad $\left(E^{\prime}\right)$ is polystable because $E^{\prime}$ is polystable. Since $\bar{f}$ is an étale covering map, an Einstein-Hermitian connection on $\operatorname{ad}\left(E^{\prime}\right)$ produces an Einstein-Hermitian connection on the direct image $\bar{f}_{*} \operatorname{ad}\left(E^{\prime}\right)$. We note that this uses the fact that the Kähler form on $Y^{\prime}$ is the pullback of the Kähler form on $X$.

Since $\bar{f}_{*} \operatorname{ad}\left(E^{\prime}\right)$ admits an Einstein-Hermitian connection, it follows that $\bar{f}_{*} \operatorname{ad}\left(E^{\prime}\right)$ is polystable. Since $\operatorname{ad}\left(E_{G}\right)$ is a direct summand of $\bar{f}_{*} \operatorname{ad}\left(E^{\prime}\right)$, we conclude that $\operatorname{ad}\left(E_{G}\right)$ is polystable.

The Einstein-Hermitian connection on $\bar{f}_{*} \operatorname{ad}\left(E^{\prime}\right)$ preserves $\operatorname{ad}\left(E_{G}\right)$, because $\operatorname{ad}\left(E_{G}\right)$ is a direct summand of $\bar{f}_{*} \operatorname{ad}\left(E^{\prime}\right)$. The connection $\nabla$ on $\operatorname{ad}\left(E_{G}\right)$ obtained this way is Einstein-Hermitian. 
Take the Einstein-Hermitian connection on $\operatorname{ad}\left(E^{\prime}\right)$ to be one given by an Einstein-Hermitian connection on $E^{\prime}$. Therefore, the Einstein-Hermitian connection on $\operatorname{ad}\left(E^{\prime}\right)$ is compatible with the Lie algebra structure of the fibers of $\operatorname{ad}\left(E^{\prime}\right)$. This implies that the above connection $\nabla$ on $\operatorname{ad}\left(E_{G}\right)$ is compatible with the Lie algebra structure of the fibers of $\operatorname{ad}\left(E_{G}\right)$. Therefore, $\nabla$ defines a connection on the principal Aut $(\operatorname{Lie}(G))$-bundle

$$
E_{\text {Aut }(\operatorname{Lie}(G))}=E_{G} \times{ }^{G} \operatorname{Aut}(\operatorname{Lie}(G))
$$

associated to $E_{G}$ for the homomorphism $G \longrightarrow \operatorname{Aut}(\operatorname{Lie}(G))$ given by the adjoint action of $G$ on $\operatorname{Lie}(G)$. This connection on $E_{\text {Aut }(\operatorname{Lie}(G))}$ given by $\nabla$ will be denoted by $\nabla_{0}$. This connection $\nabla_{0}$ is Einstein-Hermitian because $\nabla$ is so.

Define $G^{a b}:=G /[G, G]$. The quotient homomorphism $G \longrightarrow G^{a b}$ will be denoted by $q$. Let $G_{0}^{a b} \subset G^{a b}$ be the connected component containing the identity element. Let

$$
\beta: G^{a b} \longrightarrow G^{a b}
$$

be the homomorphism defined by $z \longmapsto z^{n}$, where $n$ is the order of the quotient group $G^{a b} / G_{0}^{a b}$. Note that $\beta\left(G_{0}^{a b}\right)=G_{0}^{a b}$, and the homomorphism

$$
G^{a b} / G_{0}^{a b} \longrightarrow G^{a b} / G_{0}^{a b}
$$

given by $\beta$ is the trivial homomorphism. Hence $\beta\left(G^{a b}\right)=G_{0}^{a b}$. Define

$$
\gamma:=\beta \circ q: G \longrightarrow G_{0}^{a b} .
$$

Since $G_{0}^{a b}$ is a torus, the principal $G_{0}^{a b}$-bundle $E_{G_{0}^{a b}}$ on $X$ obtained by extending the structure group of $E_{G}$ using $\gamma$ has a unique Einstein-Hermitian connection. We will denote this EinsteinHermitian connection on $E_{G_{0}^{a b}}$ by $\nabla^{\prime}$.

The connection $\nabla_{0}$ (respectively, $\nabla^{\prime}$ ) is a 1 -form on the total space $E_{\mathrm{Aut}(\operatorname{Lie}(G))}$ (respectively, $\left.E_{G_{0}^{a b}}\right)$ with values in the Lie algebra $\operatorname{Lie}(\operatorname{Aut}(\operatorname{Lie}(G)))\left(\operatorname{respectively,~} \operatorname{Lie}\left(G_{0}^{a b}\right)\right)$. Using the natural map $E_{G} \longrightarrow E_{\mathrm{Aut}(\operatorname{Lie}(G))}$ (respectively, $E_{G} \longrightarrow E_{G_{0}^{a b}}$ ), the 1-form $\nabla_{0}$ (respectively, $\nabla^{\prime}$ ) pulls back to a 1 -form on $E_{G}$ with values in $\operatorname{Lie}(\operatorname{Aut}(\operatorname{Lie}(G)))\left(\operatorname{respectively,~} \operatorname{Lie}\left(G_{0}^{a b}\right)\right)$; this 1-form on $E_{G}$ will be denoted by $\widehat{\nabla}$ (respectively, $\widehat{\nabla^{\prime}}$ ). Note that

$$
\operatorname{Lie}(G)=\operatorname{Lie}(\operatorname{Aut}(\operatorname{Lie}(G))) \oplus \operatorname{Lie}\left(G_{0}^{a b}\right) .
$$

Therefore, $\widehat{\nabla}+\widehat{\nabla^{\prime}}$ is a 1 -form on $E_{G}$ with values in the Lie algebra Lie $(G)$. It is straightforward to check that this $\operatorname{Lie}(G)$-valued 1 -form $\widehat{\nabla}+\widehat{\nabla^{\prime}}$ defines a connection on $E_{G}$. This connection on $E_{G}$ will be denoted by $\widetilde{\nabla}$.

Fix a point $x_{0} \in X$. Let $\operatorname{Aut}\left(\left(E_{G}\right)_{x_{0}}\right)$ denote the group of automorphisms of $\left(E_{G}\right)_{x_{0}}$ that commute with the action of $G$ on $\left(E_{G}\right)_{x_{0}}$. Note that $\operatorname{Aut}\left(\left(E_{G}\right)_{x_{0}}\right)$ is identified with the fiber of the adjoint bundle $\operatorname{Ad}\left(E_{G}\right)_{x_{0}}$, and it is isomorphic to $G$. Consider parallel translations of the fiber $\left(E_{G}\right)_{x_{0}}$, along loops based at $x_{0}$, with respect to the above connection $\widetilde{\nabla}$. These together produce a subgroup of $\operatorname{Aut}\left(\left(E_{G}\right)_{x_{0}}\right)$. It can be shown that this subgroup is contained in a compact subgroup of $\operatorname{Aut}\left(\left(E_{G}\right)_{x_{0}}\right)$. Indeed, this follows from the fact that the holonomies of both $\nabla_{0}$ and $\nabla^{\prime}$ are compact. Therefore, possibly taking an extension of structure group, we get a Hermitian structure on $E_{G}$. (The above subgroup of $\operatorname{Aut}\left(\left(E_{G}\right)_{x_{0}}\right)$ is a conjugate of a subgroup of $K$.) This Hermitian structure is Einstein-Hermitian because both $\nabla_{0}$ and $\nabla^{\prime}$ are so.

An Einstein-Hermitian connection on the principal $G$-bundle $E_{G} \longrightarrow X$ pulls back to an Einstein-Hermitian connection on the principal $G_{0}$-bundle $E^{\prime} \longrightarrow Y^{\prime}$. Therefore, the uniqueness of the Einstein-Hermitian connection on $E_{G}$ follows from the uniqueness of the EinsteinHermitian connection on $E^{\prime}$. To explain this, from [11, p. 24, Theorem 1] we know that a stable 
bundle has a unique Einstein-Hermitian connection, and from [6, p. 111, Theorem 3.27] we know that for any decomposition of a polystable vector bundle $F$ into a direct sum of stable vector bundles, each direct summand is preserved by any Einstein-Hermitian connection on $F$. Now apply this to the adjoint vector bundle ad $\left(E^{\prime}\right)$ and the principal $G_{0} /\left[G_{0}, G_{0}\right]$-bundle associated to $E^{\prime}$.

\section{Equivalence of stability conditions}

For a parabolic subalgebra $\mathfrak{p}$ of $\operatorname{Lie}(G)$, by $N_{G}(\mathfrak{p})$ we will denote the subgroup of $G$ that preserves $\mathfrak{p}$ by the adjoint action. In this section, by a parabolic subgroup of $G$ we will mean a group of the form $N_{G}(\mathfrak{p})$ for some parabolic subalgebra $\mathfrak{p}$. As before, by $Z_{0}(G)$ we will denote the connected component of the center of $G$ containing the identity element.

Definition 2. A principal $G$-bundle is called adjoint semistable (respectively, adjoint stable) if for all reductions to a proper parabolic subgroup $P$, and all reductions of structure group $\left.E_{P} \subset E\right|_{U}$ on a big open subset $U \subset X$, and for all strictly dominant characters $\chi: P \longrightarrow \mathbb{C}^{*}$, the associated line bundle $E_{P}(\chi)$ satisfies

$$
\left.\operatorname{deg}\left(E_{P}(\chi)\right)<0 \quad \text { (respectively, } \operatorname{deg}\left(E_{P}(\chi)\right) \leq 0\right),
$$

where the degree is calculated with respect to the Kähler form $\omega$.

A character of $P$ is called dominant if the restriction to $P_{0}:=P \cap G_{0}$ is dominant.

Recall that a reduction $\left.E_{P} \subset E\right|_{U}$ on a big open set $U \subset X$ to a parabolic subgroup $P$ is called admissible if for every nontrivial character $\chi$ of $P$ trivial on $Z_{0}(G)$, we have $\operatorname{deg}\left(E_{P}(\chi)\right)=0$.

A semistable principal $G$-bundle on $X$ is called adjoint polystable if there exists a reduction $E_{L(P)} \subset E_{G}$ to a Levi factor $L(P)$ of a parabolic subgroup $P \subset G$ such that the following two conditions hold:

- the principal $L(P)$-bundle $E_{L(P)}$ is stable, and

- the reduction of $E_{G}$ to $P$ obtained by extending the structure group of $E_{L(P)}$ to $P$ is admissible.

Lemma 2. A principal $G$-bundle $E$ on $X$ is adjoint semistable if and only if it is semistable in the sense of Definition 1.

Proof. Recall that a principal $G$-bundle $E$ on $X$ induces a principal $G_{0}$-bundle $E_{0}$ on $Y=E / G_{0}$. By Definition 1, a principal $G$-bundle $E$ is semistable if and only if the restriction of $E_{0}$ to a connected component $Y^{\prime}$ of $Y$ is semistable. This is equivalent to $\operatorname{ad}\left(E_{0}\right)$ being semistable (cf. [1, p. 214, Proposition 2.10]).

Note that $\operatorname{ad}\left(E_{0}\right)$ is isomorphic to $f^{*} \operatorname{ad}(E)$, which is $\Gamma$-equivariant. If $\operatorname{ad}\left(E_{0}\right)$ is semistable, the it is also equivariantly semistable. On the other hand, suppose that it is unstable. Its HarderNarasimhan filtration is unique, so it will be equivariant, and hence $\operatorname{ad}\left(E_{0}\right)$ will be equivariantly unstable. Therefore, $\operatorname{ad}\left(E_{0}\right)$ is semistable if and only if it is equivariantly semistable.

Taking the quotient by $\Gamma$, this is equivalent to the vector bundle $\operatorname{ad}(E)$ on $X$ being semistable. We remark that the proof of [1, p. 214, Proposition 2.10] also works for disconnected groups if we use Definition 2. So $\operatorname{ad}(E)$ is semistable if and only if $E$ is adjoint semistable.

Lemma 3. A principal $G$-bundle $E$ on $X$ is adjoint polystable if and only if it is polystable in the sense of Definition 1. 
Proof. If $E$ is polystable in the sense of Definition 1, then from Theorem 1 it follows that the adjoint vector bundle $\operatorname{ad}(E)$ is polystable. Conversely, if $\operatorname{ad}(E)$ is polystable, then $f^{*} \operatorname{ad}(E)$ is polystable because an Einstein-Hermitian connection on $\operatorname{ad}(E)$ pulls back to an EinsteinHermitian connection on $f^{*} \operatorname{ad}(E)$. If $f^{*} \operatorname{ad}(E)$ is polystable, from [1, p. 224, Corollary 3.8] we know that $E$ is polystable in the sense of Definition 1.

On the other hand, $\operatorname{ad}(E)$ is polystable if and only if $E$ is adjoint polystable; its proof is identical to the proof of [1, p. 224, Corollary 3.8].

\section{Acknowledgements}

We would like to thank B. Conrad and A. Nair for discussions. The first-named author acknowledges the support of the J.C. Bose Fellowship. The second-named author thanks the Tata Institute of Fundamental Research for the hospitality during a visit where part of this work was done. This work was partly funded by the grant MTM2010-17389 and ICMAT Severo Ochoa project SEV-2011-0087 of the Spanish Ministerio de Economía y Competitividad.

\section{References}

[1] Anchouche B., Biswas I., Einstein-Hermitian connections on polystable principal bundles over a compact Kähler manifold, Amer. J. Math. 123 (2001), 207-228.

[2] Atiyah M.F., Complex analytic connections in fibre bundles, Trans. Amer. Math. Soc. 85 (1957), $181-207$.

[3] Behrend K.A., Semi-stability of reductive group schemes over curves, Math. Ann. 301 (1995), 281-305.

[4] Biswas I., Subramanian S., Semistability and finite maps, Arch. Math. (Basel) 93 (2009), 437-443.

[5] Gómez T.L., Langer A., Schmitt A.H.W., Sols I., Moduli spaces for principal bundles in large characteristic, in Teichmüller Theory and Moduli Problem, Ramanujan Math. Soc. Lect. Notes Ser., Vol. 10, Ramanujan Math. Soc., Mysore, 2010, 281-371, math.AG/0506511.

[6] Kobayashi S., Differential geometry of complex vector bundles, Publications of the Mathematical Society of Japan, Vol. 15, Princeton University Press, Princeton, NJ, 1987.

[7] Koszul J.L., Lectures on fibre bundles and differential geometry, Tata Institute of Fundamental Research Lectures on Mathematics, Vol. 20, Tata Institute of Fundamental Research, Bombay, 1960, available at http: //www.math.tifr.res.in/ publ/ln/tifr20.pdf.

[8] Ramanathan A., Moduli for principal bundles, in Algebraic Geometry (Proc. Summer Meeting, Univ. Copenhagen, Copenhagen, 1978), Lecture Notes in Math., Vol. 732, Springer, Berlin, 1979, 527-533.

[9] Ramanathan A., Moduli for principal bundles over algebraic curves. I, Proc. Indian Acad. Sci. Math. Sci. 106 (1996), 301-328.

[10] Ramanathan A., Moduli for principal bundles over algebraic curves. II, Proc. Indian Acad. Sci. Math. Sci. 106 (1996), 421-449.

[11] Ramanathan A., Subramanian S., Einstein-Hermitian connections on principal bundles and stability, J. Reine Angew. Math. 390 (1988), 21-31.

[12] Springer T.A., Linear algebraic groups, Progress in Mathematics, Vol. 9, 2nd ed., Birkhäuser Boston Inc., Boston, MA, 1998. 